Research Article

\title{
Further Properties of 1- and 2-Dimensional U- and W-Convexity and Fixed Point of Nonexpansive Mappings in Banach Spaces $X$ and $X^{*}$
}

\author{
Ji Gao \\ Department of Mathematics, Community College of Philadelphia, Philadelphia, Pennsylvania 19130-3991, USA \\ Correspondence should be addressed to Ji Gao; jgao@ccp.edu
}

Received 7 February 2021; Accepted 26 April 2021; Published 4 May 2021

Academic Editor: Zoran Mitrovic

Copyright (c) 2021 Ji Gao. This is an open access article distributed under the Creative Commons Attribution License, which permits unrestricted use, distribution, and reproduction in any medium, provided the original work is properly cited.

In this paper, we further studied properties of the modulus of $n$-dimensional $U$-convexity and the modulus of $n$-dimensional $U$-flatness when $n=1$ (2-dimensional character) and $n=2$ (3-dimensional character). The new properties of these moduli are investigated, and the relationships between these moduli and other geometric parameters of Banach spaces are studied. Some results on fixed point theory for nonexpansive mappings and normal structure in Banach spaces are obtained.

\section{Introduction}

Let $X$ be a real Banach space with the dual space $X^{*}$. Let $B(X)=\{x \in X:\|x\| \leq 1\}$ and $S(X)=\{x \in X:\|x\|=1\}$ be the unit ball and the unit sphere of $X$, respectively. Let $X^{*}$ be the dual space of $X$. Let $\nabla_{x} \subset S\left(X^{*}\right)$ denote the set of norm 1 supporting functionals of $x \in S(X)$.

Brodskii and Mil'man [1] introduced the following geometric concepts in 1948.

Definition 1. Let $X$ be a Banach space. A nonempty bounded and convex subset $K$ of $X$ is said to have normal structure if, for every convex subset $C$ of $K$ that contains more than one point, there is a point $x_{0} \in C$ such that

$$
\sup \left\{\left\|x_{0}-y\right\|: y \in C\right\}<\operatorname{diam} C .
$$

A Banach space $X$ is said to have

(a) Normal structure if every bounded convex subset of $X$ has normal structure.

(b) Weak normal structure if every weakly compact convex set $K$ of $X$ has normal structure. (c) Uniform normal structure if there exists $0<c<1$ such that, for every bounded closed convex subset $C$ of $K$ that contains more than one point, there is a point $x_{0} \in C$ such that

$$
\sup \left\{\left\|x_{0}-y\right\|: y \in C\right\}<c \cdot \operatorname{diam} C
$$

Remark 1. The following facts are known:

(a) Uniform normal structure $\Rightarrow$ normal structure $\Rightarrow$ weak normal structure

(b) For a reflexive spaces, normal structure $\Leftrightarrow$ weak normal structure

Kirk [2] proved that if a Banach space $X$ has weak normal structure, then it has weak fixed point property, that is, every nonexpansive mapping from a weakly compact and convex subset of $X$ to itself has a fixed point.

Let $\mathbb{N}$ be the set of all natural numbers and $n \in \mathbb{N}$.

For two sets of vectors $\left\{x_{i}\right\}_{i=1}^{n+1} \subseteq X$ and $\left\{f_{i}\right\}_{i=2}^{n+1} \subseteq X^{*}$, the $(n+1) \times(n+1)$ matrix, 


$$
\left[\begin{array}{cccc}
1 & 1 & \cdots & 1 \\
\left\langle x_{1}, f_{2}\right\rangle & \left\langle x_{2}, f_{2}\right\rangle & \cdots & \left\langle x_{n+1}, f_{2}\right\rangle \\
\vdots & \vdots & \ddots & \vdots \\
\left\langle x_{1}, f_{n+1}\right\rangle & \left\langle x_{2}, f_{n+1}\right\rangle & \cdots & \left\langle x_{n+1}, f_{n+1}\right\rangle
\end{array}\right],
$$

is denoted by $m\left(x_{1}, x_{2}, \ldots, x_{n+1} ; f_{2}, f_{3}, \ldots, f_{n+1}\right)$ [3].

Gao and Saejung [3] introduced the concept of volume by the convex hull of $x_{1}, x_{2}, \ldots, x_{n+1}$ in $X$ of

$v\left(x_{1}, x_{2}, \ldots, x_{n+1}\right):=\sup \left\{\operatorname{det} m\left(x_{1}, x_{2}, \ldots, x_{n+1} ; f_{2}, f_{3}, \ldots, f_{n+1}\right)\right\}$,

where the supremum is taken over all $f_{i} \in \nabla_{x_{i}}$, where $i=2,3, \ldots, n+1$.
Definition 2 (see [3]). Let $v_{X}^{n}=\sup \left\{v\left(x_{1}, x_{2}, \ldots, x_{n+1}\right): x_{1}\right.$, $\left.x_{2}, \ldots x_{n+1} \in S(X)\right\}$ be the upper bound of all $n$-dimensional volume in $X$.

The following result was proved [3].

Proposition 1. For a Banach space $X$ with $\operatorname{dim}(X)>n$, we have $v_{X}^{n} \geq 2$.

Gao and Saejung introduced the concept of the modulus of $n$-dimensional $U$-convexity of $X$ as follows.

Definition 3 (see [3]). Let $X$ be a Banach space. Define

$$
U_{X}^{n}(\varepsilon)=\inf \left\{1-\frac{1}{n+1}\left\|x_{1}+x_{2}+\ldots+x_{n+1}\right\|: \begin{array}{c}
x_{1}, x_{2}, \ldots, x_{n+1} \in S(X), \\
v\left(x_{1}, x_{2}, \ldots, x_{n+1}\right) \geq \varepsilon
\end{array}\right\}
$$

where $0 \leq \varepsilon \leq v_{X}^{n}$ is the modulus of $n$-dimensional $U$-convexity of $X$.

The following results were proved in [3] too.

\section{Theorem 1}

(a) $U_{X}^{n}(\varepsilon)$ is an increasing and continuous function in $\left[0, v_{X}^{n}\right)$ for any $n \in \mathbb{N}$.

(b) If $X$ is a Banach space with $U_{X}^{n}(1)>0$ for some $n \in \mathbb{N}$, then $X$ is super-reflexive.

(c) If $X$ is a Banach space with $U_{X}^{n}(1)>0$ for some $n \in \mathbb{N}$, then $X$ has uniform normal structure

The following results were proved in [4].

Theorem 2. If $X$ is a Banach space with $U_{X}^{n}\left(2 n+1 / 2^{n}\right)>1-$ $1 / n+1$ for some $n \in \mathbb{N}$ and $n$ is even, then $X$ is reflexive.
Let $n=2$, and we have the following.

Corollary 1. If $X$ is a Banach space with $U_{X}^{2}(5 / 4)>2 / 3$, then $X$ is reflexive.

The following results were also proved in [4].

Theorem 3. If $X$ is a Banach space with $U_{X}^{2}(5 / 4)>2 / 3$ and $U_{X}^{2}(5 / 2)>5 / 6$, then $X$ has uniform normal structure.

Gao and Saejung also introduced the concept of the modulus of $n$-dimensional $U$-flatness as follows.

Definition 4 (see [5]). Let $X$ be a Banach space. Define

$$
W_{X}^{n}(\varepsilon)=\sup \left\{1-\frac{1}{n+1}\left\|x_{1}+x_{2}+\ldots+x_{n+1}\right\|: \begin{array}{c}
x_{1}, x_{2}, \ldots, x_{n+1} \in S(X), \\
v\left(x_{1}, x_{2}, \ldots, x_{n+1}\right) \leq \varepsilon
\end{array}\right\}
$$

where $0 \leq \varepsilon \leq v_{X}^{n}$ is the modulus of $n$-dimensional $U$-flatness of $X$.

Remark 2. (a) The name of the modulus of $U$-flatness is defined by comparing with the name of the modulus of $U$-convexity of $X$. (b) $U_{X}^{n}(\varepsilon) \leq W_{X}^{n}(\varepsilon)$ in $\left[0, v_{X}^{n}\right)$.

The following results were proved in [5] too.

\section{Theorem 4}

(a) $W_{X}^{n}(\varepsilon)$ is an increasing and continuous function in $\left[0, v_{X}^{n}\right)$ for any $n \in \mathbb{N}$. (b) If $X$ is a Banach space with $W_{X}^{1}(5 / 3)<2 / 3$, or $W_{X}^{2}(7 / 9)<2 / 3$, then $X$ is reflexive.

(c) If $X$ is a Banach space with $W_{X}^{n}\left(2 n+1 / 2^{n}\right)<$ $1-1 / n+1$, where $n \in \mathbb{N}$, then $X$ is super-reflexive.

(d) Suppose that $X$ is a Banach space satisfying one of the following conditions:

(i) $W_{X}^{n}(1)<1-1 / n+1$, for some $n \in \mathbb{N}$ with $n \geq 3$

(ii) $W_{X}^{n}\left(2 n+1 / 2^{n}\right)<1-1 / n+1$, for $n=1$ or $n=2$

Then, $X$ has uniform normal structure.

Recently, Gabeleh introduced concepts of pointwise cyclic relatively nonexpansive mapping and weak proximal normal 
structure for an extension of geometric property of normal structure. Some interesting results are obtained there [6].

In this paper, we studied further properties of the modulus of $n$-dimensional $U$-convexity and the modulus of $n$-dimensional $U$-flatness when $n=1$ (2-dimensional character) and $n=2$ (3-dimensional character). The new properties of these moduli are investigated and the relationships between these moduli and other geometric parameters of Banach spaces are studied. Some results on fixed point theory for nonexpansive mappings and normal structure in Banach spaces are obtained.

\section{Main Results}

Lemma 1 (Bishop-Phelps-Bollobás, see [7]). Let $X$ be a Banach space, and let $0<\varepsilon<1$. Given $z \in B(X)$ and $h \in S\left(X^{*}\right)$ with $1-\langle z, h\rangle<\varepsilon^{2} / 4$, then there exist $y \in S(X)$ and $g \in \nabla_{y}$ such that $\|y-z\|<\varepsilon$ and $\|g-h\|<\varepsilon$.

Since $\langle z, h\rangle$ is a continuous function in norm for $z \in X$ and norm for $h \in X^{*}$, Lemma 1 can be stated as follows.
Lemma 2. Let $X$ be a Banach space, and let $0<\varepsilon<1$. Given $z \in S(X)$ and $h \in B\left(X^{*}\right)$ with $1-\langle z, h\rangle<\varepsilon^{2} / 4$, then there exist $y \in S(X)$ and $g \in \nabla_{y}$ such that $\|y-z\|<\varepsilon$ and $\|g-h\|<\varepsilon$.

Theorem 5 (see [8]). Let $X$ be a Banach space. Then, $X$ is not reflexive if and only if, for any $0<\delta<1$, there are a sequence $\left\{x_{n}\right\} \subseteq S(X)$ and a sequence $\left\{f_{n}\right\} \subseteq S\left(X^{*}\right)$ such that

(a) $\left\langle x_{m}, f_{n}\right\rangle=\delta$ whenever $n \leq m$

(b) $\left\langle x_{m}, f_{n}\right\rangle=0$ whenever $n>m$

Theorem 6. If $X$ is a Banach space with $U_{X^{*}}^{1}(2)>3 / 4$, or $W_{X^{*}}^{1}(2)<1 / 2$, then $X$ is reflexive.

Proof. Suppose that $X$ is not reflexive. Let $0<\delta<1$ be given. Let $f_{1} \in S\left(X^{*}\right),-f_{1}+f_{2} / 2 \in B\left(X^{*}\right)$, and $x_{1} \in S\left(X^{* *}\right)$, $-x_{2} \in S\left(X^{* *}\right)$. Then, we have

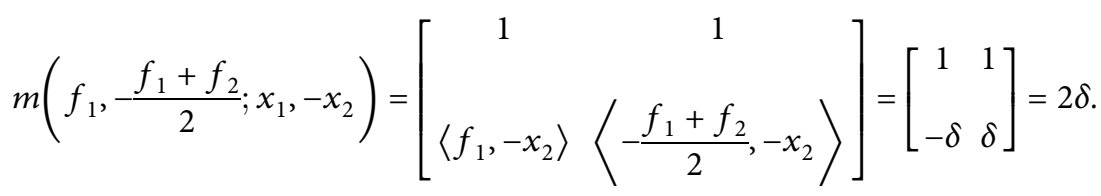

We also have

$\frac{1}{4} \leq\left\langle x_{1}, \frac{1}{2}\left(f_{1}-\frac{f_{1}+f_{2}}{2}\right)\right\rangle \leq \frac{1}{2}\left\|f_{1}-\frac{f_{1}+f_{2}}{2}\right\|=\frac{1}{2}\left\|\frac{f_{1}-f_{2}}{2}\right\| \leq \frac{1}{2}$.

So,

$$
\frac{1}{2} \leq 1-\frac{\left\|f_{1}-f_{1}+f_{2} / 2\right\|}{2} \leq \frac{3}{4} .
$$

Since $\delta$ can be arbitrarily close to 1 , by using Lemma 2 and the definition of $U_{X}^{1}(\varepsilon)$ and $W_{X}^{1}(\varepsilon)$, we have if $U_{X^{*}}^{1}(2)>3 / 4$, or $W_{X^{*}}^{1}(2)<1 / 2$, then $X$ is reflexive.
Theorem 7. If $X$ is a Banach space with $U_{X^{*}}^{2}(2 / 3)>2 / 3$, or $W_{X^{*}}^{2}(2 / 3)<5 / 9$, then $X$ is reflexive.

Proof. Suppose that $X$ is not reflexive. Let $0<\delta<1$ be given. Let $f_{1} \in S\left(X^{*}\right),-f_{1}+f_{2} / 2 \in B\left(X^{*}\right), f_{1}+f_{2}+f_{3} / 3 \in B\left(X^{*}\right)$, and $x_{1} \in S\left(X^{* *}\right),-x_{2} \in S\left(X^{* *}\right)$, and $x_{3} \in S\left(X^{* *}\right)$.

Then, we have

$$
\begin{aligned}
& m\left(f_{1},-\frac{f_{1}+f_{2}}{2}, \frac{f_{1}+f_{2}+f_{3}}{3} ; x_{1},-x_{2}, x_{3}\right)
\end{aligned}
$$

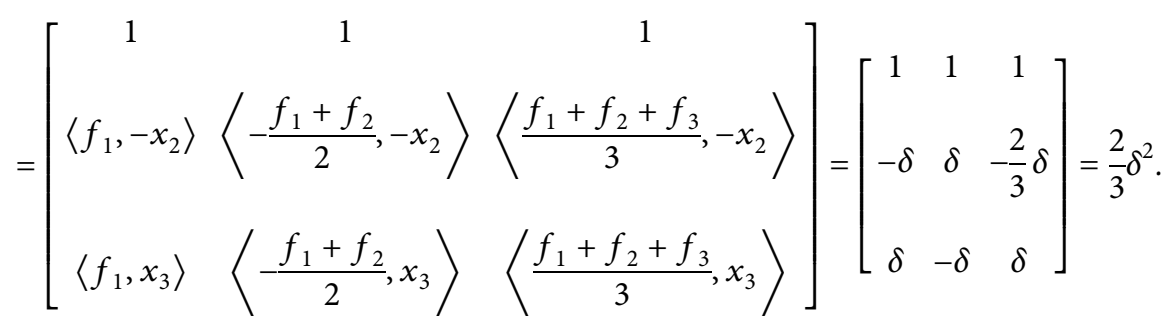

We also have 


$$
\begin{aligned}
& \frac{1}{3}\left\|f_{1}-\frac{f_{1}+f_{2}}{2}+\frac{f_{1}+f_{2}+f_{3}}{3}\right\|=\frac{\left\|5 f_{1}-f_{2}+2 f_{3}\right\|}{18} \leq \frac{4}{9} \\
& \frac{1}{3}\left\|f_{1}-\frac{f_{1}+f_{2}}{2}+\frac{f_{1}+f_{2}+f_{3}}{3}\right\|=\frac{\left\|5 f_{1}-f_{2}+2 f_{3}\right\|}{18} \geq\left\langle\frac{5 f_{1}-f_{2}+2 f_{3}}{18}, x_{3}\right\rangle=\frac{1}{3} .
\end{aligned}
$$

We have

$$
\frac{5}{9} \leq 1-\frac{1}{3}\left\|f_{1}-\frac{f_{1}+f_{2}}{2}+\frac{f_{1}+f_{2}+f_{3}}{3}\right\| \leq \frac{2}{3} .
$$

Since $\delta$ can be arbitrarily close to 1 , by using Theorem 2.2 and the definition of $U_{X}^{2}(\varepsilon)$ and $W_{X}^{2}(\varepsilon)$, we have if $U_{X^{*}}^{2}(2 / 3)>2 / 3$, or $W_{X^{*}}^{2}(2 / 3)<5 / 9$, then $X$ is reflexive.

The following result refers to a Banach space with weak* sequentially compact unit ball of the dual. Notice that this property is satisfied by reflexive or separable Banach spaces and by those that admit an equivalent smooth norm.

Lemma 3 (see [9]). If $X$ is a Banach space with $B\left(X^{*}\right)$ weak sequentially compact and fails to have weak normal structure, then, for any $\varepsilon>0$, there are sequence $\left\{x_{n}\right\} \subseteq S(X)$ and sequence $\left\{f_{n}\right\} \subseteq S\left(X^{*}\right)$ such that (a) $\left|\left\|x_{i}-x_{j}\right\|-1\right|<\varepsilon$, whenever $i \neq j$

(b) $\left\langle x_{i}, f_{i}\right\rangle=1$, whenever $1 \leq i \leq \infty$

(c) $\left|\left\langle x_{j}, f_{i}\right\rangle\right|<\varepsilon$, whenever $i \neq j$

(d) $\left\|f_{i}-f_{j}\right\|>2-\varepsilon$, whenever $i \neq j$

Theorem 8. If $X$ is a Banach space with $U_{X^{*}}^{1}(2)>1 / 2$, or $W_{X^{*}}^{1}(2)<1 / 2$, then $X$ has weak normal structure.

Proof. Suppose that $X$ does not have weak normal structure. Let $\varepsilon>0$ be given. Since $2 \geq\left\|f_{2}-f_{1}\right\| \geq\left\langle f_{2}-f_{1}, x_{2}-\right.$ $x_{1}>/ 1+\varepsilon \geq 2-2 \varepsilon / 1+\varepsilon$, we have $1-\varepsilon / 1+\varepsilon \leq\left\|f_{2}-f_{1} / 2\right\| \leq 1$.

Let $f_{1} \in S\left(X^{*}\right), f_{2}-f_{1} / 2 \in B\left(X^{*}\right)$; and $x_{1} \in S\left(X^{* *}\right)$ and $x_{2}-x_{1} /\left\|x_{2}-x_{1}\right\| \in S\left(X^{* *}\right)$. Then, we have

$$
m\left(f_{1}, \frac{f_{2}-f_{1}}{2} ; x_{1}, \frac{x_{2}-x_{1}}{\left\|x_{2}-x_{1}\right\|}\right)=\left[\left\langle f_{1}, \frac{x_{2}-x_{1}}{\left\|x_{2}-x_{1}\right\|}\right\rangle\left\langle\frac{f_{2}-f_{1}}{2}, \frac{x_{2}-x_{1}}{\left\|x_{2}-x_{1}\right\|}\right\rangle\right]=\left[\begin{array}{c}
1 \\
\frac{-1+a \varepsilon}{\left\|x_{2}-x_{1}\right\|} \frac{1+b \varepsilon}{\left\|x_{2}-x_{1}\right\|}
\end{array}\right]=2+c \varepsilon
$$

where $a, b$, and $c$ are constants and $\varepsilon$ is arbitrarily small. We also have

$$
\begin{aligned}
\frac{1}{2}-\mathrm{d} \varepsilon & \leq\left\langle\frac{x_{1}-x_{2}}{\left\|x_{1}-x_{2}\right\|}, \frac{1}{2}\left(\frac{f_{1}-f_{2}}{2}\right)\right\rangle \\
& \leq \frac{1}{2}\left\|f_{1}+\frac{f_{2}-f_{1}}{2}\right\|=\frac{1}{2}\left\|\frac{f_{1}+f_{2}}{2}\right\| \leq \frac{1}{2},
\end{aligned}
$$

where $d$ is a constant.

So,

$$
\frac{1}{2} \leq 1-\frac{1}{2}\left\|f_{1}+\frac{f_{2}-f_{1}}{2}\right\|=\frac{1}{2}+\mathrm{d} \varepsilon
$$

By using Theorem 2.2 and the definition of $U_{X}^{1}(\varepsilon)$ and $W_{X}^{1}(\varepsilon)$, we have if $U_{X^{*}}^{1}(2)>1 / 2$, or $W_{X^{*}}^{1}(2)<1 / 2$, then $X$ has weak normal structure.

Theorem 9. If $X$ is a Banach space with $U_{X^{*}}^{2}(9 / 4)>5 / 6$, or $W_{X^{*}}^{2}(9 / 4)<2 / 3$, then $X$ has weak normal structure.

Proof. Suppose that $X$ does not have weak normal structure. Let $\varepsilon>0$ be given.

Let $f_{1} \in S\left(X^{*}\right), f_{2}-f_{1} / 2 \in B\left(X^{*}\right), f_{3}-f_{2} / 2 \in B\left(X^{*}\right)$; and $x_{1} \in S\left(X^{* *}\right), x_{2}-x_{1} /\left\|x_{2}-x_{1}\right\| \in S\left(X^{* *}\right)$, and $x_{3}-x_{2} /$ $\left\|x_{3}-x_{2}\right\| \in S\left(X^{* *}\right)$. Then, similar to the proof of Theorem 8 , we have 


$$
\begin{aligned}
& m\left(f_{1}, \frac{f_{2}-f_{1}}{2}, \frac{f_{3}-f_{2}}{2} ; x_{1}, \frac{x_{2}-x_{1}}{\left\|x_{2}-x_{1}\right\|}, \frac{x_{3}-x_{2}}{\left\|x_{3}-x_{2}\right\|}\right) \\
& =\left[\begin{array}{c}
\left\langle f_{1}, \frac{x_{2}-x_{1}}{\left\|x_{2}-x_{1}\right\|}\right\rangle\left\langle\frac{f_{2}-f_{1}}{2}, \frac{x_{2}-x_{1}}{\left\|x_{2}-x_{1}\right\|}\right\rangle\left\langle\frac{f_{3}-f_{2}}{2}, \frac{x_{2}-x_{1}}{\left\|x_{2}-x_{1}\right\|}\right\rangle \\
\left\langle f_{1}, \frac{x_{3}-x_{2}}{\left\|x_{3}-x_{2}\right\|}\right\rangle\left\langle\frac{f_{2}-f_{1}}{2}, \frac{x_{3}-x_{2}}{\left\|x_{3}-x_{2}\right\|}\right\rangle\left\langle\frac{f_{3}-f_{2}}{2}, \frac{x_{3}-x_{2}}{\left\|x_{3}-x_{2}\right\|}\right\rangle
\end{array}\right]=\left[\begin{array}{ccc}
1 & 1 & 1 \\
-1+a \varepsilon & 1+b \varepsilon & -\frac{1}{2}+c \mathcal{E} \\
d \varepsilon & -\frac{1}{2}+e \varepsilon & 1+f \mathcal{E}
\end{array}\right]=\frac{9}{4}+g \varepsilon,
\end{aligned}
$$

where $a, b, c, d, e, f$, and $g$ are constants and $\varepsilon$ is arbitrarily small.

We also have

$$
\begin{aligned}
\frac{1}{6}-\varepsilon & \leq\left\langle x_{1}, \frac{f_{1}+f_{3}}{6}\right\rangle \leq \frac{1}{3}\left\|f_{1}+\frac{f_{2}-f_{1}}{2}+\frac{f_{3}-f_{2}}{2}\right\| \\
& =\frac{\left\|f_{1}+f_{3}\right\|}{6} \leq \frac{1}{3},
\end{aligned}
$$

and we have

$$
\frac{2}{3} \leq 1-\frac{\left\|f_{1}+f_{3}\right\|}{6} \leq \frac{5}{6}+\varepsilon .
$$

By using Theorem 2.2 and the definition of $U_{X}^{2}(\varepsilon)$ and $W_{X}^{2}(\varepsilon)$, we have if $U_{X^{*}}^{2}(9 / 4)>5 / 6$, or $W_{X^{*}}^{2}(9 / 4)<2 / 3$, then $X$ has weak normal structure.

Definition 5 (see $[10,11])$. Let $X$ and $Y$ be Banach spaces. We say that $Y$ is finitely representable in $X$ if, for any $\varepsilon>0$ and any finite dimensional subspace $N \subseteq Y$, there is an isomorphism $T: N \longrightarrow X$ such that, for any $y \in N$, $(1-\varepsilon)\|y\| \leq\|T y\| \leq(1+\varepsilon)\|y\|$.

We say that $X$ is super-reflexive if any space $Y$ which is finitely representable in $X$ is reflexive.

Theorem 10. Suppose that $X$ is a Banach space satisfying one of the following conditions:
(a) $U_{X^{*}}^{1}(2)>3 / 4$
(b) $U_{X^{*}}^{2}(2 / 3)>2 / 3$
(c) $W_{X^{*}}^{1}(2)<1 / 2$
(d) $W_{X^{*}}^{2}(2 / 3)<5 / 9$

Then, $X$ is super-reflexive.

Proof. The proof of this theorem follows from the fact that if a Banach space $Y$ is finitely representable in $X$, then $U_{Y}^{n}(\varepsilon)=$ $U_{X}^{n}(\varepsilon)$ and $W_{Y}^{n}(\varepsilon)=W_{X}^{n}(\varepsilon)$, for any $n \in \mathbb{N}$ and for any $\varepsilon \in v_{X}^{n}$.

We consider the uniform normal structure. To discuss this result, let us recall the concept of the "ultra"-technique.

Let $\mathscr{F}$ be a filter of an index set $I$, and let $\left\{x_{i}\right\}_{i \in I}$ be a subset in a Hausdorff topological space $X,\left\{x_{i}\right\}_{i \in I}$ is said to converge to $x$ with respect to $\mathscr{F}$, denoted by $\lim _{\mathscr{F}} x_{i}=x$, if, for each neighborhood $U$ of $x,\left\{i \in I: x_{i} \in U\right\} \in \mathscr{F}$. A filter $\mathcal{U}$ on $I$ is called an ultrafilter if it is maximal with respect to the ordering of the set inclusion. An ultrafilter is called trivial if it is of the form $\left\{A: A \subseteq I, i_{0} \in A\right\}$ for some $i_{0} \in I$. We will use the fact that if $\mathcal{U}$ is an ultrafilter, then

(i) For any $A \subseteq I$, either $A \subseteq U$ or $I-A \subseteq U$

(ii) If $\left\{x_{i}\right\}_{i \in I}$ has a cluster point $x$, then $\lim _{\mathcal{U}} x_{i}$ exists and equals to $x$

Let $\left\{X_{i}\right\}_{i \in I}$ be a family of Banach spaces and let $l_{\infty}\left(I, X_{i}\right)$ denote the subspace of the product space equipped with the norm $\left\|\left(x_{i}\right)\right\|=\sup _{i \in I}\left\|x_{i}\right\|<\infty$.

Definition 6 (see $[12,13])$. Let $\mathcal{U}$ be an ultrafilter on $I$ and let $N_{U}=\left\{\left(x_{i}\right) \in l_{\infty}\left(I, X_{i}\right): \lim _{\mathcal{U}}\left\|x_{i}\right\|=0\right\}$. The ultraproduct of $\left\{X_{i}\right\}_{i \in I}$ is the quotient space $l_{\infty}\left(I, X_{i}\right) / N_{\mathcal{U}}$ equipped with the quotient norm.

We will use $\left(x_{i}\right)_{\mathscr{U}}$ to denote the element of the ultraproduct. It follows from remark (ii) above and the definition of quotient norm that

$$
\left\|\left(x_{i}\right)_{\mathcal{U}}\right\|=\lim _{\mathcal{U}}\left\|x_{i}\right\|
$$

In the following, we will restrict our index set $I$ to be $\mathbb{N}$, the set of natural numbers, and let $X_{i}=X, i \in \mathbb{N}$, for some Banach space $X$. For an ultrafilter $\mathcal{U}$ on $\mathbb{N}$, we use $X_{\mathscr{U}}$ to denote the ultraproduct. Note that if $\mathscr{U}$ is nontrivial, then $X$ can be embedded into $X_{\mathscr{U}}$ isometrically.

Lemma 4 (see [13]). Suppose that $\mathcal{U}$ is an ultrafilter on $\mathbb{N}$ and $X$ is a Banach space. Then, $\left(X^{*}\right)_{\mathcal{U}} \cong\left(X_{\mathscr{U}}\right)^{*}$ if and only if $X$ is super-reflexive; in this case, the mapping $J$ defined by

$$
\left\langle\left(x_{i}\right)_{\mathscr{U}}, J\left(\left(f_{i}\right)_{\mathcal{U}}\right)\right\rangle=\lim _{\mathcal{U}}\left\langle x_{i}, f_{i}\right\rangle, \quad \text { for all }\left(x_{i}\right)_{\mathscr{U}} \in \mathrm{X}_{\mathcal{U}},
$$

which is the canonical isometric isomorphism from $\left(X^{*}\right)_{\mathscr{U}}$ onto $\left(X_{U}\right)^{*}$.

Theorem 11. Let $X$ be a super-reflexive Banach space. Then, for any nontrivial ultrafilter $\mathcal{U}$ on $\mathbb{N}$ and for all $n \in \mathbb{N}$ and $\varepsilon>0$, we have $U_{X_{\mathcal{U}}}^{n}(\varepsilon)=U_{X}^{n}(\varepsilon)$, and $W_{X_{\mathcal{U}}}^{n}(\varepsilon)=W_{X}^{n}(\varepsilon)$.

Proof. The proof is the same as the proof of Theorem 2.17 in [3]. 
Lemma 5 (see [14]). If $X$ is a super-reflexive Banach space, then $X$ has uniform normal structure if and only if $X_{U}$ has normal structure.

Theorem 12. Suppose that $X$ is a Banach space satisfying one of the following conditions:

(a) $U_{X^{*}}^{1}(2)>3 / 4$

(b) $U_{X^{*}}^{2}(2 / 3)>2 / 3$ and $z_{X^{*}}^{1}(2)>1 / 2$, or $U_{X^{*}}^{2}(2 / 3)>2 / 3$ and $W_{X^{*}}^{1}(2)<1 / 2$, or $U_{X^{*}}^{2}(2 / 3)>2 / 3$ and $U_{X^{*}}^{2}(9 / 4)$ $>5 / 6$, or $U_{X^{*}}^{2}(2 / 3)>2 / 3$ and $W_{X^{*}}^{2}(9 / 4)<2 / 3$

(c) $W_{X^{*}}^{1}(2)<1 / 2$

(d) $W_{X^{*}}^{2}(2 / 3)<5 / 9$ and $U_{X^{*}}^{1}(2)>1 / 2$, or $W_{X^{*}}^{2}(2 / 3)<$ $5 / 9$ and $W_{X^{*}}^{1}(2)<1 / 2$, or $U_{X^{*}}^{2}(2 / 3)<5 / 9$ and $U_{X^{*}}^{2}$ $(9 / 4)>5 / 6$, or $W_{X^{*}}^{2}(2 / 3)<5 / 9$ and $W_{X^{*}}^{2}(9 / 4)<2 / 3$

Then, $X$ has uniform normal structure.

Proof. The results (a) and (c) follow directly from Theorem 8, Theorems 10 and 11, and Lemma 5. The results (b) and (d) follow directly from Theorems 9,10 , and 11 and Lemma 5.

\section{Data Availability}

No data were used to support this study.

\section{Conflicts of Interest}

The authors declare that they have no conflicts of interest.

\section{References}

[1] M. S. Brodskiì and D. P. Mil'man, "On the center of a convex set. (Russian),” Doklady Akad. Nauk SSSR (N.S.), vol. 59, pp. 837-840, 1948.

[2] W. A. Kirk, "A fixed point theorem for mappings which do not increase distances," The American Mathematical Monthly, vol. 72, pp. 1004-1006, 1965.

[3] J. Gao and S. Saejung, "The $n$-dimensional U-convexity and geometry of banach spaces," Journal of Fixed Point Theory, vol. 16, no. 2, pp. 381-392, 2015.

[4] J. Gao, "Modulus of 2-dimensional U-convexity and the geometry of Banach spaces," Journal of Nonlinear and Convex Analysis, vol. 20, no. 10, pp. 2041-2051, 2019.

[5] J. Gao and S. Saejung, "U-flatness and non-expansive mappings in Banach spaces," Journal of the Korean Mathematical Society, vol. 54, no. 2, pp. 493-506, 2017.

[6] M. Gabeleh, "A characterization of proximal normal structure via proximal diametral sequences," Journal of Fixed Point Theory and Applications, vol. 19, no. 4, pp. 2909-2925, 2017.

[7] B. Bollobás, "An extension to the theorem of Bishop and Phelps," Bulletin of the London Mathematical Society, vol. 2, no. 2, pp. 181-182, 1970.

[8] R. C. James, "Weakly compact sets," Transactions of the American Mathematical Society, vol. 113, no. 1, p. 129, 1964.

[9] S. Saejung and J. Gao, "Fixed points, normal structure and moduli of semi-UKK, semi-NUC and semi-UKK* spaces," Journal of the Egyptian Mathematical Society, vol. 23, no. 1, pp. 113-118, 2015.

[10] M. M. Day, Normed Linear Spaces Ergebnisse der Mathematik und ihrer Grenzgebiete, Band 21, Springer, New York, NY, USA, 3rd edition, 1989.
[11] J. Diestel, Geometry of Banach Spaces-Selected Topics, Springer-Verlag, Berlin, Germany, 2006.

[12] D. Dacunha-Castelle and J. Krivine, "Application des ultraproduits à l'étude des espaces et des algèbres de Banach," Studia Mathematica, vol. 41, no. 3, pp. 315-334, 1972.

[13] B. Sims, "Ultra"-Techniques in Banach Space Theory, Vol. 60, Queen's University, Kingston, Ontario, Canada, 1982.

[14] M. A. Khamsi, "Uniform smoothness implies super-normal structure property," Nonlinear Analysis: Theory, Methods \& Applications, vol. 19, no. 11, pp. 1063-1069, 1992. 